\title{
PEMLIF - a tale of two stories
}

\section{Raja K. Kutty, Balakrishnan Sreemathyamma Sunilkumar, Anilkumar Peethambaran, Sourabh Kumar Jain, Shailesh Kumar}

Department of Neurosurgery, Government Medical College, Thiruvananthapuram, India

Correspondence to: Raja K. Kutty, M.S, M.Ch. Department of Neurosurgery, Government Medical College, Thiruvananthapuram, Kerala 695011, India. Email: drrajakkutty@gmail.com.

\begin{abstract}
Intervertebral disc prolapse is a common cause of both acute and chronic low back pain. This can result in differential grades of motor and sensory disturbances in lower limbs and even cauda equina syndrome (CES). The neurological manifestations are a result of compression of the spinal cord and roots ventrally in the spinal canal due to the prolapsed disc. However, neurologic deficits owing to compression of the spinal cord dorsally as a result of migration of sequestrated disc fragments are very rare. We present two different cases of back pain with severe neurologic deficit referred to us within a short span of one month. Among them, one had long standing history of back pain while other had none. Both patients were investigated and found to have a sequestrated disc fragment which had dorsally migrated to the epidural space and was causing severe compression of the spinal cord. They underwent laminectomy and removal of the sequestrated fragment. However, they were only partially relieved of their motor and bladder weakness. We present our cases to highlight this rare form of lumbar epidural intervertebral disc migration, as well as enumerate the diagnostic challenges and discuss the differential diagnosis and treatment.
\end{abstract}

Keywords: Posterior epidural migration; lumbar disc; cauda equina

Submitted Dec 13, 2016. Accepted for publication Apr 12, 2017.

doi: 10.21037/jss.2017.08.07

View this article at: http://dx.doi.org/10.21037/jss.2017.08.07

\section{Introduction}

Posterior migration of the intervertebral disc was first reported as 'posterior rotation of the annulus fibrosis' by Vincenzo Lombardi in 1973 (1). Quoted as PEMLIF an acronym for posterior epidural migration of lumbar intervertebral disc fragment, it is a very rare phenomenon with less than fifty cases reported in the literature. Migration of the sequestrated segment is not only limited to the lumbar region, but has also been reported in the thoracic $(2)$ as well as the cervical $(3,4)$ segments. However, this is most commonly seen in the lumbar spinal segments and least in the cervical region. The various ligamentous structures in the posterior spinal canal make the anatomy of this space unique. These ligamentous structures are distinctively anchored in this space to hinder the free passage of the sequestrated segments of the disc material to the dorsal epidural space. This obstruction is reinforced posteriorly by the strong posterior longitudinal ligament
(PLL). The acute neurologic symptoms produced by the dorsal compression of the spinal cord by the epidurally migrated sequestrated disc fragment are very rare. Most of the cases reported previously have been on a background of a recurring back pain before manifestation of overt neurological signs. We present two distinct cases of posterior epidural migration of lumbar disc fragment that we encountered in our unit within a span of one month.

\section{Case presentation}

\section{Case 1}

A 48-year-old man was who had no previous history of back ache was involved in a strenuous activity wherein he had to lift weight of about $25 \mathrm{~kg}$. Following this, he suddenly felt excruciating pain in the lower back radiating down to both his buttocks and knees. After sometime he felt weakness of both lower limbs and could not walk 

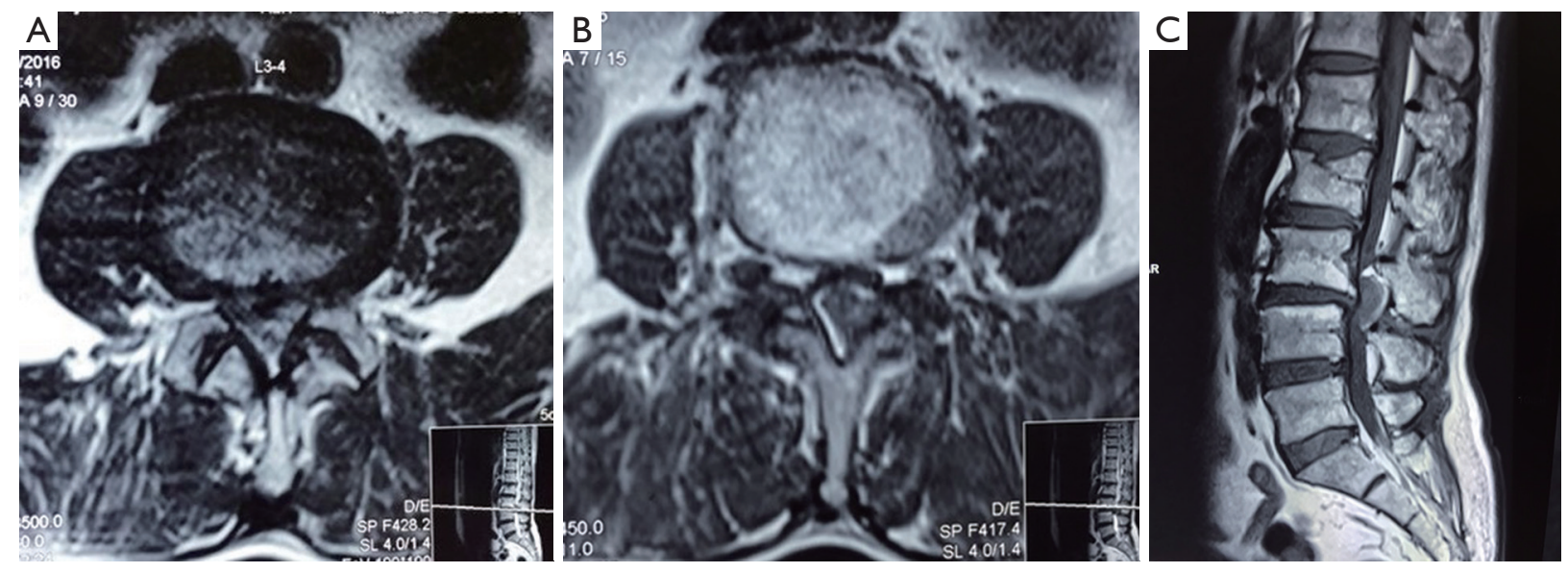

Figure $1 \mathrm{MRI}$ spine. (A) T1W axial image at L3-4 level showing gross obliteration of the lumbar spinal canal by the migrated disc; (B) T2W axial image at L3-4 level showing the obliteration of the cerebrospinal fluid space due to compression by the migrated disc fragment; (C) sagittal T1W image showing narrowing of the disc at L3-4 level with the migrated disc fragment at the posterior epidural space. MRI, magnetic resonance imaging; T1W, T1-weighted; T2W, T2-weighted.

without support. Additionally, he reported urinary and bowel incontinence. He was taken to a local hospital from where he was referred to our services after five days. On examination, his straight leg raising was positive, bulk and tone were normal, and he had power of $3 / 5$ in his hip and knee and grade $0 / 5$ power in both ankle dorsiflexion and plantarflexion. Reflexes were absent in the lower limbs. He had saddle anesthesia in his lower limbs and sensations below L2 were decreased. Magnetic resonance imaging (MRI) findings were suggestive of intervertebral disc prolapse in the region of L3-4 level (Figure 1). It was decided to perform an emergency decompression at that level. At surgery, a laminectomy was performed at the L3 level. Even after the ligamentum flavum was excised via Kerrison punch, the dura was not visible. An operating microscope was brought into the field and it was visualized that the entire spinal canal was filled with the extruded disc fragment. After removing the sequestrated segments, the dura was seen to be severely compressed anteriorly and the decompression restored the flow of the CSF in the caudal dural sac. The rent in the annulus was visualized under the microscope after retracting the dura and the space was inspected for any residual disc fragments with the help of a hook. Post-operatively, the patient had immediate relief from his pain. His weakness of his limbs gradually improved to grade $4 / 5$ in hip and knee but the power in his ankle did not show drastic improvement. At discharge, he had grade $2 / 5$ in his ankle dorsiflexion and extension and he had urinary incontinence persisting along with bowel complaints. At follow up of 3 months he did not gain control over his bowel or bladder but he improved his power by one grade in his ankle dorsiflexion and plantarflexion.

\section{Case 2}

A 49-year-old male, who had a history of low back ache over the last 2 years, presented with acute aggravation of pain since the last 2 weeks. He had numbness in both legs and difficulty in walking. There was no history of trauma. On examination, straight leg examination was positive. Bulk and tone was normal and he had power of grade $4 / 5$ in hips bilaterally, knees - left $4 / 5$, right $5 / 5$, ankle was of grade $0 / 5$ dorsiflexion and plantarflexion was $2 / 5$ in foot bilaterally. There was reduced sensation below L3 dermatome. He had urinary incontinence. MRI of the lumbosacral spine revealed degenerative changes in the lumbar vertebrae in addition to a soft tissue lesion $2.1 \mathrm{~cm} \times 1.1 \mathrm{~cm}$ in the spinal canal at L3 level posterior to the thecal sac. It was hypointense on T1, slightly hyperintense on T2 and showed mild peripheral enhancement of a possibly migrated disc (Figure 2). He was taken up for surgery. At surgery, L3 partial lamina was drilled and it was possible to visualize the disc fragment. The fragment could be retrieved after minimally enlarging the size of the laminectomy (Figures 3,4). The laminectomy was then extended bilaterally and the thickened ligamentum flavum was excised to give maximum decompression to the underlying dura (Figure 5). Postoperatively the patient recovered well from his pain. 

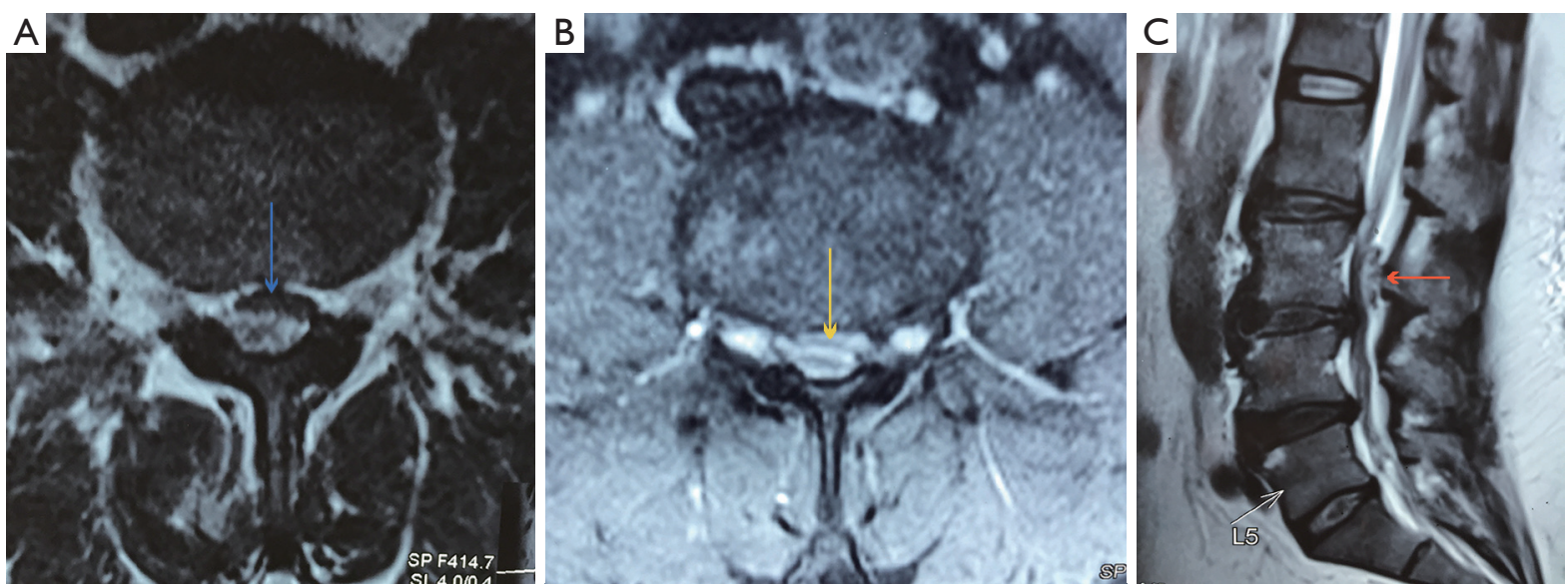

Figure 2 MRI spine. (A) T1W axial plain image at L3-4 level showing compression of the dura by the extruded disc fragment (slender blue arrow); (B) T1W axial with contrast showing peripheral contrast enhancement of the extruded disc fragment (slender yellow arrow); (C) T1W Sagittal image showing compression of the dural sac by the extruded disc fragment (red arrow). MRI, magnetic resonance imaging; T1W, T1-weighted.

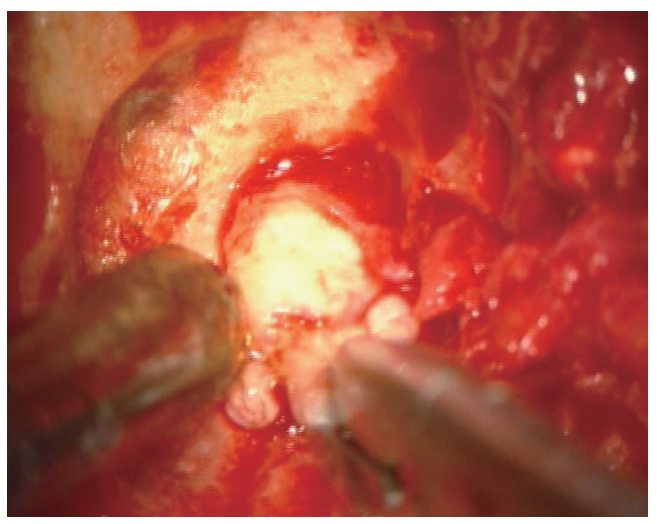

Figure 3 Intraoperative photograph showing part of the extruded disc being teased out through the partial laminectomy at L3.

There was only partial recovery from his bladder symptoms and the power of his lower limbs improved to grade 2/5, for ankle dorsiflexion and plantarflexion. At follow-up of 3 months, this further improved to grade $3 / 5$ for both ankle dorsiflexion and plantarflexion while recovery for his bladder remained unchanged.

\section{Discussion}

In the above two illustrations, we present two distinct cases PEMLIF with different histories.

The anatomic peculiarity of the posterior spinal canal makes this form of migration of disc very rare. There are

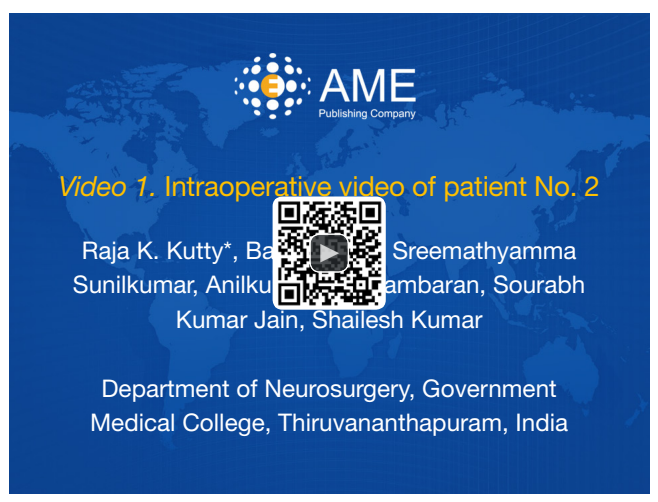

Figure 4 Intraoperative video of patient No. 2. Standing on the left side of the patient. The spinous process of $\mathrm{L} 3$ vertebrae has already been removed. Part of the Lamina just lateral to spinous process of $\mathrm{L} 3$ vertebrae near the midline towards left side is being drilled. The sequestrated disc fragment was beginning to be visualized. It is gently being teased out from the laminectomy site (5). Available online: http://www.asvide.com/articles/1749

many ligamentous connections between the dura and PLLs. These are the posterior median septum also known as the septum posticum, the lateral peridural membrane and finally the midline and lateral dural ligaments of Hoffmann. These connections are a limiting factor in determining the path of migration of the intervertebral disc (6). The lateral peridural membrane resists the posterolateral migration of the extruded disc fragment. Hence in cases of PEMLIF 

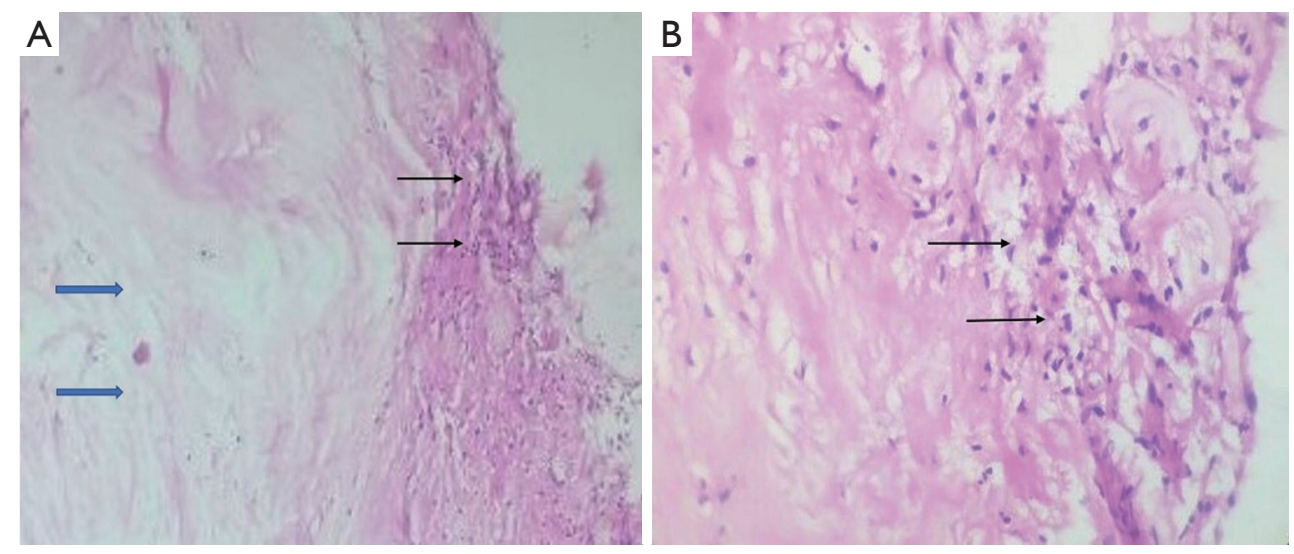

Figure 5 Histopathology slides of the extruded disc material. (A) Photomicrograph, H\&E, magnification: 40x. Degenerative disc material (stout blue arrows). Invading polymorphonuclear cells with granulation tissue (slender black arrows) in the periphery of the disc material; (B) photomicrograph, H\&E, magnification: 400×. Invading polymorphonuclear cells seen in the periphery of the disc margins. H\&E, hemotoxylin and eosin.

it is implied that the fragment has pierced the septum posticum as well as the lateral peridural membrane to reach the posterior epidural space. In addition, the disc fragment may migrate in a cephalad or caudal direction. Cephalad migration is common with lateral disc whereas caudal migration is common with central discs (7). According to some authors this happens depending upon the site of the tear of the annulus. A tear at the superior endplate leads to a cephalad migration compared to a tear in the inferior direction (8). In another landmark article, none of the factors were found to influence the direction of migration of the disc fragment in the lumbar canal (9). PEMLIF as quoted in the literature is very rare. A recent review of literature by Akhaddar et al. found that the total number of cases was only about 41 (10). This generally occurs in the setting of a degenerating spine pathology and patients usually give a previous history of backache like our second patient. However, our first patient did not have such a history. To our knowledge, this is the second instance wherein a patient presents with gross neurological findings as a sequelae to PEMLIF without a history of back pain (11). The PLL, dura, fat, epidural vessels and the nerve roots can act as a barrier for the migration of the disc in the posterior direction. The posterior migration of the disc is associated with varying clinical findings and has been associated with cauda equina syndrome (CES). The upper lumbar vertebrae, particularly L3 and L4, are mostly involved in the cases pertaining to posterior epidural migration of disc in the literature. A recent study found an incidence of $39.2 \%$ for posterior migrations at these levels. According to them, the peculiar anatomical orientation between the disc and the nerve root at these levels predisposes to this phenomenon (12). The intervertebral disc at this segment is more horizontal and this association between the nerve root and the disc may render it less obstructive for epidural migration; this reason has been cited by other authors (13). In both our patients, the migrated discs were opposite to the L3 vertebral body.

The radiology of the PEMLIF deserves special mention as they are often mistaken for other lesions. Herniated disc fragments sequestrated in the posterior epidural space may simulate other commonly occurring pathologies in the spinal canal like epidural abscesses (14), hematoma (14), tumors (15) and facet cysts (16). The likelihood of a diagnosis of posterior migration of disc is commonly less considered by reporting radiologists. In a recent review of six cases of posteriorly migrated discs by Zarrabian et al., the favored diagnoses of seasoned radiologists were epidural hematoma, epidural abscess and synovial cyst (17). Herniated discs are hypointense on T1 weighted and hyperintense in $80 \%$ of T2 weighted images. This T2 hyperintensity reveals the abundance of water content within the disc and thus could also explain the enhanced softness and motility of the extruded fragment, which helps it reach the posterior epidural space.

The posteriorly migrated disc fragment gets surrounded by the epidural fat which mounts an inflammatory response leading to the formation of granulation tissue $(6,12,18)$. The creeping of polymorphonuclear cells and granulation tissue can be visualized in the periphery of 
the disc material on the histopathology slide. It is this granulation tissue that surrounds the disc and is usually seen as contrast enhancement on MRI films. This type of peripheral enhancement was seen in our second patient. This was confirmed in the histology specimen. There have been reports of such ring-enhancing masses in the dorsal epidural space as a sequelae to PEMLIF (19). Routine MRI comprises of $5 \mathrm{~mm}$ slices in the sagittal cuts. In cases with a high index of suspicion of PEMLIF, thin slices of $2 \mathrm{~mm}$ cuts can be requested of the radiologist beforehand. This can detect the thin connections, if at all present, between the parent disc and the sequestrated segment.

CES is a serious sequelae to disc herniations. Its incidence is about 1 in 33,000 to 100,000 and it occurs in $2 \%$ of all lumbar disc herniations (20). CES with bilateral foot drop is very rare. CES with dorsal disc migration is scarcely reported in the literature with fewer than 22 cases reported (10).

The recovery period after surgery for CES is variable. However, multiple authors opine that early surgery does bring in good results. Shapiro et al. showed patients had good recovery if operated upon within 48 hours (21). The outcome in patients with CES and PEMLIF is better than CES with their ventral counterparts (10). To achieve a maximum decompression after removal of the sequestrated segment in both cases, we did a bilateral laminectomy of the involved segment along with the removal of the ligamentum flavum. However, there was a delay of about one week in achieving this goal owing to the late referral in both our patients. Hence both our patients did not have very good postoperative recovery.

\section{Conclusions}

PEMLIF is a rare phenomenon. The exact pathomechanisms have yet to be deciphered. The early detection of this variety of disc should be kept in mind for patients who present with acute neurologic symptoms. The elucidation of history is key to the diagnosis. Radiologists should keep a low threshold for diagnosis in a background of a typical history and be prompted to obtain thin sagittal cuts which may show a continuity of disc fragments. Early decompression should be the goal of surgery as restoration of bladder and CES have been shown to be better than with compression with discs ventrally.

\section{Acknowledgements}

None.

\section{Footnote}

Conflicts of Interest: The authors have no conflicts of interest to declare.

Ethical Statement: As this is a retrospective case report this does not warrant an ethical committee clearance from the institution.

\section{References}

1. Lombardi V. Lumbar spinal block by posterior rotation of anulus fibrosus. Case report. J Neurosurg 1973;39:642-7.

2. Miyakoshi N, Hongo M, Kasukawa Y, et al. Posteriorly migrated thoracic disc herniation: a case report. J Med Case Rep 2013;7:41.

3. Tofuku K, Koga H, Kawabata N, et al. Dorsally sequestered cervical disc herniation. Spine (Phila Pa 1976) 2007;32:E837-40.

4. Srinivasan US, Kumar GS, Mahesha KB. Posterior epidural migration of sequestrated cervical disc fragment: case series. Asian Spine J 2011;5:220-7.

5. Kutty RK, Sunilkumar BS, Peethambaran A, et al. Intraoperative video of patient No. 2. Asvide 2017;4:433. Available online: http://www.asvide.com/articles/1749

6. Dösoğlu M, Is M, Gezen F, et al. Posterior epidural migration of a lumbar disc fragment causing cauda equina syndrome: case report and review of the relevant literature. Eur Spine J 2001;10:348-51.

7. Ebeling U, Reulen HJ. Are there typical localisations of lumbar disc herniations? A prospective study. Acta Neurochir (Wien) 1992;117:143-8.

8. Song KJ, Lee KB. Non-traumatic acute myelopathy due to cervical disc herniation in contiguous two-level disc spaces: a case report. Eur Spine J 2005;14:694-7.

9. Schellinger D, Manz HJ, Vidic B, et al. Disk fragment migration. Radiology 1990;175:831-6.

10. Akhaddar A, El-Asri A, Boucetta M. Posterior epidural migration of a lumbar disc fragment: a series of 6 cases. J Neurosurg Spine 2011;15:117-28.

11. Ju JH, Kim HW, Jung CK, et al. Sudden onset of cauda equina syndrome resulting from posterior migration of lumbar herniated disc without significant previous neurological signs. Korean J Spine 2012;9:281-4.

12. Turan Y, Yilmaz T, Gocmez C, et al. Posterior Epidural Migration of a Sequestered Lumbar Intervertebral Disc Fragment. Turk Neurosurg 2017;27:85-94.

13. Sengoz A, Kotil K, Tasdemiroglu E. Posterior epidural 
migration of herniated lumbar disc fragment. J Neurosurg Spine 2011;14:313-7.

14. Derincek A, Ozalay M, Sen O, et al. Posterior epidural mass: can a posteriorly migrated lumbar disc fragment mimic tumour, haematoma or abscess? Acta Orthop Belg 2009;75:423-7.

15. Ekşi MS, Yener U, Akakin A, et al. Posterior epidural disc herniation at L3-L4 mimicking a spinal tumor: a case report. J Neurosurg Sci 2010;54:71-6.

16. Yoo YS, Ju CI, Kim SW, et al. Posterior epidural migration of an extruded lumbar disc mimicking a facet cyst: a case report. Korean J Spine 2015;12:12-4.

17. Zarrabian MM, Diehn FE, Kotsenas AL, et al. Dorsal Lumbar Disc Migrations with Lateral and Ventral Epidural Extension on Axial MRI: A Case Series and Review of

Cite this article as: Kutty RK, Sunilkumar BS, Peethambaran A, Jain SK, Kumar S. PEMLIF-a tale of two stories. J Spine Surg 2017;3(3):498-503. doi: 10.21037/jss.2017.08.07
Literature. AJNR Am J Neuroradiol 2016. [Epub ahead of print].

18. Teufack SG, Singh H, Harrop J, et al. Dorsal epidural intervertebral disk herniation with atypical radiographic findings: case report and literature review. J Spinal Cord Med 2010;33:268-71.

19. Toktaş ZO, Yılmaz B, Konya D, et al. Posterior epidural migration of lumbar disc fragment as an unusual ringenhancing mass. Spine J 2016;16:e31-2.

20. Tamburrelli FC, Genitiempo M, Bochicchio M, et al. Cauda equina syndrome: evaluation of the clinical outcome. Eur Rev Med Pharmacol Sci 2014;18:1098-105.

21. Shapiro S. Cauda equina syndrome secondary to lumbar disc herniation. Neurosurgery 1993;32:743-6; discussion 746-7. 